\title{
Identification and Quantification of Genomic Repeats and Sample Contamination in Assemblies of 454 Pyrosequencing Reads
}

\author{
Alexander J. Nederbragt, Trine Ballestad Rounge, Kyrre L. Kausrud, and Kjetill S. Jakobsen \\ Department of Biology, Centre for Ecological and Evolutionary Synthesis (CEES), \\ University of Oslo, P.O. Box 1066 Blindern, 0316 Oslo, Norway
}

Correspondence should be addressed to Kjetill S. Jakobsen, kjetill.jakobsen@bio.uio.no

Received 26 May 2009; Revised 28 September 2009; Accepted 5 November 2009

Academic Editor: Nick Loman

Copyright ( $(2010$ Alexander J. Nederbragt et al. This is an open access article distributed under the Creative Commons Attribution License, which permits unrestricted use, distribution, and reproduction in any medium, provided the original work is properly cited.

Contigs assembled from 454 reads from bacterial genomes demonstrate a range of read depths, with a number of contigs having a depth that is far higher than can be expected. For reference genome sequence datasets, there exists a high correlation between the contig specific read depth and the number of copies present in the genome. We developed a sequence of applied statistical analyses, which suggest that the number of copies present can be reliably estimated based on the read depth distribution in de novo genome assemblies. Read depths of contigs of de novo cyanobacterial genome assemblies were determined, and several high read depth contigs were identified. These contigs were shown to mainly contain genes that are known to be present in multiple copies in bacterial genomes. For these assemblies, a correlation between read depth and copy number was experimentally demonstrated using real-time PCR. Copy number estimates, obtained using the statistical analysis developed in this work, are presented. Percontig read depth analysis of assemblies based on 454 reads therefore enables de novo detection of genomic repeats and estimation of the copy number of these repeats. Additionally, our analysis efficiently identified contigs stemming from sample contamination, allowing for their removal from the assembly.

\section{Introduction}

During assembly of shotgun datasets using the 454 (Newbler) assembly program, reads stemming from regions in the genome that are repeated, that is, present in multiple copies with a high degree of similarity, "collapse" into a single contig $[1,2]$. Collapsed contigs start and end with the parts of reads that extend into the collapsed alignment. Specifically, reads that align partly within and partly outside a repeated region are divided between two contigs (in contrast to some other assembly programs, e.g., phrap [3], that do not divide reads between contigs). Thus, each collapsed contig only represents a repeated part of the genome including all (parts of) reads derived from the repeated regions. Consequently, these contigs have a higher read depth, where read depth is defined as the number of bases from all the reads used to assemble the contig, divided by the contig consensus length.

Studying contigs of a shotgun assembly of a cyanobacterial genome (Planktothrix rubescens NIVA CYA 98), we discovered that certain genes were likely present in several copies in the genome [4]. The number of copies of these genes seemed to be correlated to the number of reads used to assemble the contigs. In this study, we investigate whether this phenomenon is a general property of 454 shotgun assemblies. We hypothesize that, due to the even distribution of 454 reads over the genome [1,5-8], the per-contig read depth should be linearly proportional to the number of genomic copies present in the genome.

To this end, we have analyzed whole genome shotgun assemblies of sequence read datasets obtained with 454 technology, focusing on bacterial genome and BAC datasets for which no pairwise information was present. An analysis of consensus contigs based on read depth, that is, the number of sequence reads that represent (cover) a base in the consensus contig, is presented. We also present a statistical approach to de novo estimate the number of copies of these regions present in the genome. By taking the per-contig read depth into account, de novo assemblies based on 454 reads 
can yield additional information on the genome of study regarding repeated sequence regions.

Additionally, we find that if we assemble genomes based on DNA samples that include some level of contamination, read depth analysis can be used to effectively identify, quantify, and remove the contaminant.

\section{Methods}

2.1. Sequencing Datasets. Escherichia coli str. K-12 substr. MG1655 GS FLX reads were taken from a sample run accompanying the GS FLX Data Analysis Software Manual (version 1.1.03, December 2007). The GS FLX sample run consisting of only shotgun data was used. There were 547,055 GS FLX reads, totaling $\sim 127.1$ million bases (average read length 232 bases) in the dataset. The annotated reference sequence was taken from GenBank, accession number NC_000913 [9].

The Porphyromonas gingivalis W83 GS FLX reads were obtained from the NCBI Short Read Archive [10] under accession number SRA001027. The dataset consisted of 510,032 reads, totaling $\sim 116.4$ million bases (average read length 228 bases). Genbank accession number AE015924 [11] was used as annotated reference sequence.

All other datasets were obtained using the GS FLX (Roche/454) at the Ultra-high Throughput Sequencing Platform of the Centre for Ecological and Evolutionary Synthesis (CEES), Department of Biology, University of Oslo, except for part of the P. rubescens NIVA CYA98 dataset, which was sequenced at Roche, Penzburg. The standard protocol for GS FLX shotgun sequencing was followed; no paired end data was used for any of the 454 read datasets.

The sequencing of $P$. rubescens NIVA CYA98 is described elsewhere [4]. The dataset consisted of 570,912 reads, totaling 149.6 million bases (average length 262 bases). The sequencing of $A$. flos-aquae strain 10E6 is described in Stüken et al. [12]. 683,403 reads, totaling 153.4 M bases (average length 224 bases) were available for this strain.

The bacterial genomes studied had GC percentages as follows: E. coli: 50.8\%; P. gingivalis: 48.4\%; P. rubescens: $39.5 \%$; A. flos-aquae: $38.2 \%$.

The read datasets for BACs $184 \mathrm{H} 23$ and 114L13 were kindly provided by Unni Grimholt, CEES, Department of Biology, University of Oslo. The datasets consisted of 74,826 reads (12.1 $\mathrm{M}$ bases) and 55,269 reads (8.8 $\mathrm{M}$ bases), respectively.

2.2. Assembly, Mapping and Output Parsing. All datasets were assembled using Newbler (version 1.1.03) [13] with standard parameter settings, and the optional ace-file for small genomes as additional output file. The ace file is generated by Newbler in a format that follows the specifications of the phrap assembly program [3] for genome assembly, and contains information on all aligned reads used to make the contigs. Ace files were parsed, using a custom PERL script, to generate metrics on the assembly, that is, contig statistics, number of reads used, and so forth, and on the contigs, that is, read depth, percentage GC, and so forth. The script was further used to split the assembly into contigs based on length or read depth.

Linear regression analyses were performed and graphs were plotted using Origin 8 (OriginLab, Northampton, USA), and the statistical package $R$ version 2.8.1 [14].

\subsection{Statistics of Read Depth Distribution and Copy Number} Estimation. We developed a method for assessing the probabilistic relationship between read depth and the number of repeats. The read depth of independent, nonrepeated sequences approaches a Poisson process, but the existence of some repeated sequences within each contig and the fact that some nucleotide sequences are read more frequently than others cause the mean: variance ratio for single-nucleotide read depths to diverge from the $1: 1$ assumption of the Poisson distribution. Hence, we use robust, non-parametric methods to avoid biases. A robust measure of read depth ( $\lambda$ ) for contig $i$ is assumed to be the median $\left(\lambda_{m, i}\right)$, with the mean to be found between the quantiles $q_{l}$ and $q_{u}$ of the nucleotide read depths $\left(\lambda_{q l, i}, \lambda_{q u, i}\right)$ and most likely to be within one standard deviation of the median.

From the resulting empirical distribution of $\lambda_{m, i}$ 's, we pick out the tallest peak of the kernel density as representing the mean read depth of nonrepeated sequences, under the assumption that the bacterial genome is relatively sparse in repeated sequences. Treating this peak as a nonparametric density distribution, we assume that the best estimate $\theta$ of the mean read depth of nonrepeated sequences is found within the range of values covered by a proportion $\delta$ of its relative maximum probability. Furthermore, we assume that most contigs are repeated an integer number of times. This is implemented numerically, estimating $\theta$ as the value within the range defined by $\delta$ that minimizes the sum of square distances between the estimated number of repeats for each contig, $\lambda_{i} \theta^{-1}$, and their closest integers. Median, upper, and lower confidence intervals (CIs) for the average number of times contig $i$ repeated in the genome is thus found as $\lambda_{m, i} \theta^{-1}, \lambda_{q u, i} \theta_{L}^{-1}$ and $\lambda_{q l, i} \theta_{U}^{-1}$, respectively, where $\theta_{L}$ and $\theta_{U}$ are the lower and upper limits for $\theta$ as defined by $\delta$ and the empirical kernel density. Using $\delta=0.05, q_{l}=0.025$, and $q_{u}=0.975$ was seen to result in almost no errors in deciding between single-copy and repeated sequences in the known E.coli and $P$. gingivalis genomes (i.e., for a singlecopy contig, the upper limit of the empirical CI not being above $1.5 x$, and similarly, for a repeated contig, the lower limit of the empirical CI not being below $1.5 \mathrm{x}$ ). These settings seem to reflect a fairly good trade-off between precision and confidence. Due to varying degrees of degree of overlap between confidence intervals, while a fairly good estimate can be made, there will always be a trade-off between false positives and false negatives when distinguishing single from multiple copy numbers.

The analysis method was implemented as script for the statistical package $R$ [14]. The 454 AlignmentInfo.tsv generated by the Newbler assembly program was used as input for this script. This file contains, among other information, for each position in each contig the read depth of that position. The script, as well as relevant files for the E. coli 
and $P$. gingivalis assemblies, is available from the following website: http://www.sequencing.uio.no/services/scripts/.

2.4. Real-Time PCR. Contigs with a range of read depths were selected randomly from the assemblies. PCR primers with identical $\mathrm{Tm}$, generating $\sim 200 \mathrm{bp}$ PCR products were designed using Vector NTI (Invitrogen, Carlsbad, USA) and Primer3 [15]. BLAST searches against the assemblies were used to check that the primers were unique to the contig template. Average read depth at the sites of primer hybridization was calculated from the assembly alignment info files. Supplementary Table I (in Supplementary Material available online at doi:10.1155/2010/782465) gives an overview of the primers designed.

As template, the same DNA isolate, as was used for the 454 sequencing, was taken for both $P$. rubescens and $A$. flos-aqua. PCRs with four different 10-fold dilutions of the template were made to test PCR-efficiency. The PCR setup included HotGoldStar enzyme and qPCR Core kit for SYBR Green I (Eurogentec, Liege, Belgium) using a Lightcycler 480 (Roche, Basel, Switzerland). All reactions were performed in duplicate.

2.5. Taxonomic Profiling of Contigs Using MEGAN. Contigs, minimum length $500 \mathrm{bp}$ for the P. rubescens and A. flosaquae, and $100 \mathrm{bp}$ for $\mathrm{BAC}$ assemblies, were compared to the nonredundant NCBI protein database using BLASTX [16], at the University of Oslo Bioportal (www.bioportal.uio.no). Settings were: Matrix: BLOSUM62, Gap Penalties: Existence: 11, Extension: 1, Maximum $E$-value 10; the alignments of the 25 best hits were recovered as a text file. This output file was imported into the program MEGAN, version 2 beta9 [17]. The bit score cutoff chosen for the P. rubescens and A. flos-aquae contigs was 100 , for the BAC contigs 30 (bit scores represent alignment quality). Trees were collapsed to the Family taxonomic level, with "Reads summarized" (the sum of all the reads assigned to that node and all nodes below it) shown for each node.

\section{Results and Discussion}

3.1. Per Contig Read Depth of 454 Read Assemblies and Genomic Copies. We have chosen two bacterial genomes for which both 454 reads and high quality annotated reference genomes were available, Escherichia coli $\mathrm{K}-12$ [9] and Porphyromonas gingivalis W83 [11], as "model systems" for our study. The read datasets, obtained from the NCBI Short Read Archive [10], were used to explore the read distribution among contigs generated by assembling the reads.

Both GS FLX datasets were assembled, using the 454 Newbler assembler with default settings. The assembly for the E. coli dataset resulted in 100 contigs of at least $500 \mathrm{bp}$. For each contig, the per-contig read depth was determined. This per-contig read depth indicates, on average for all positions in a contig, how many times that base was covered by a read; this is sometimes referred to as "coverage". However, coverage is also used instead of oversampling (or redundancy), which is defined as the number of bases sequenced divided by genome size. The distribution of the per-contig read depths for the contigs of the E. coli assembly is shown in Figure 1(a). The read depth distribution peaks between 26 and 29x (Figure 1(a)). As the genome was sequenced to an oversampling level (or redundancy) of 27.4x ( 127.1 million bases sequenced and genome size $\sim 4.6 \mathrm{Mbp}$ ), contigs showing an approximate Poisson distribution around this number were expected. However, there was also a number of contigs with a higher read depth, up to $267 \mathrm{x}$.

A similar per-contig read depth pattern was observed for the assembly of the $P$. gingivalis GS FLX reads, which were assembled into 124 contigs of $500 \mathrm{bp}$ or more. Figure 1(b) shows that the per-contig read depth distribution for this assembly peaks around 40 to $60 \mathrm{x}$, corresponding to $49.7 \mathrm{x}$ oversampling for this genome ( $\sim 116.4$ million bases, genome size $\sim 2.3 \mathrm{Mbp}$ ). Again, this assembly also showed a number of contigs with higher read depths, up to $531 x$ (Figure 1(b)).

Using the annotated, high quality reference genomes available for E.coli K12 [9, 18] and P. gingivalis [11], BLASTN [16] was used to compare the contig sequences of both datasets with the corresponding reference genome (maximum $E$ value $10^{-16}, 97 \%$ or more identity at the nucleotide level, thereby allowing for a few mismatches). Based on the BLAST results, for each contig the number of hits in the reference genome was calculated by summing the lengths of the High-scoring Segment Pairs (the portion of the contig sequence that aligned to the reference genome) for each hit, and dividing that by the contig length. The results are shown in Figures 1(c) and 1(d) for the E. coli and P. gingivalis assemblies, respectively. Linear regression analysis showed a high correlation ( $R$-squared values 0.995 and 0.984 , resp.) between the contig read depth and the number of BLAST hits in the reference genome. These results indicate that contig read depth is indeed proportional to the number of copies present in the genome with a very high correlation.

For both genomes, Sanger reads are available. We assembled these reads, using Newbler to make sure sequences from repeated regions would collapse into single contigs with higher read depth. When the per-contig read depth analysis comparison with number of blast hits to the reference genome was repeated for these assemblies, a lower correlation was found compared to the assemblies using 454 reads ( $R$-squared values 0.897 and 0.871 for E. coli and $P$. gingivalis, resp.). This is a result of the less even distribution of the Sanger reads across the genome $[1,5-$ 8]. In addition, it has been reported that especially repeated sequences show a particularly low cloning efficiency and therefore are underrepresented in Sanger read datasets [19], a fact that contributed to reduced correlation between read depth and copy number for Sanger based assemblies. Bailey et al. [20] observed a strong correlation when comparing the number of whole genome shotgun Sanger reads mapped to $5 \mathrm{~kb}$ segments of the human genome with known diploid copy number. However, the genomic regions studies were masked for repeat sequences in that study.

Making use of annotations of the reference genomes, we analyzed which genomic regions the high read depth contigs of the GS FLX assemblies were derived from. Supplementary 


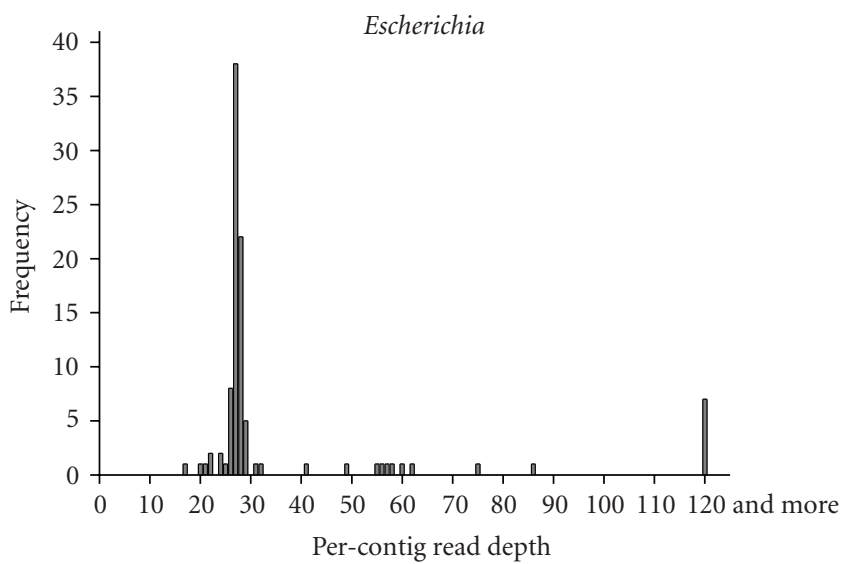

(a)

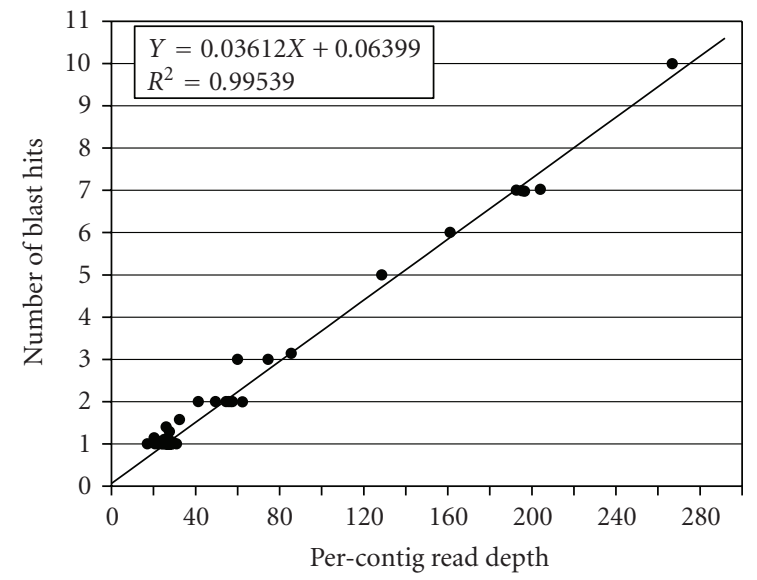

(c)

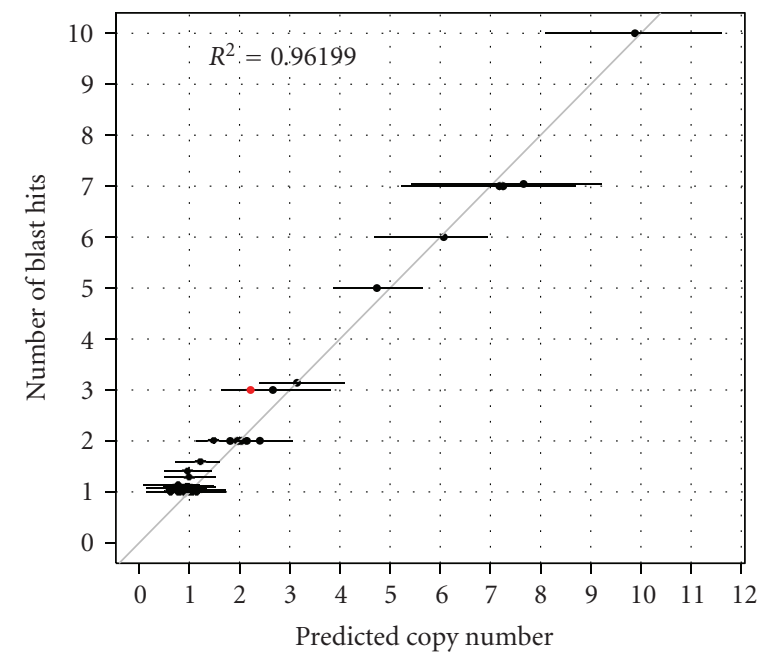

(e)

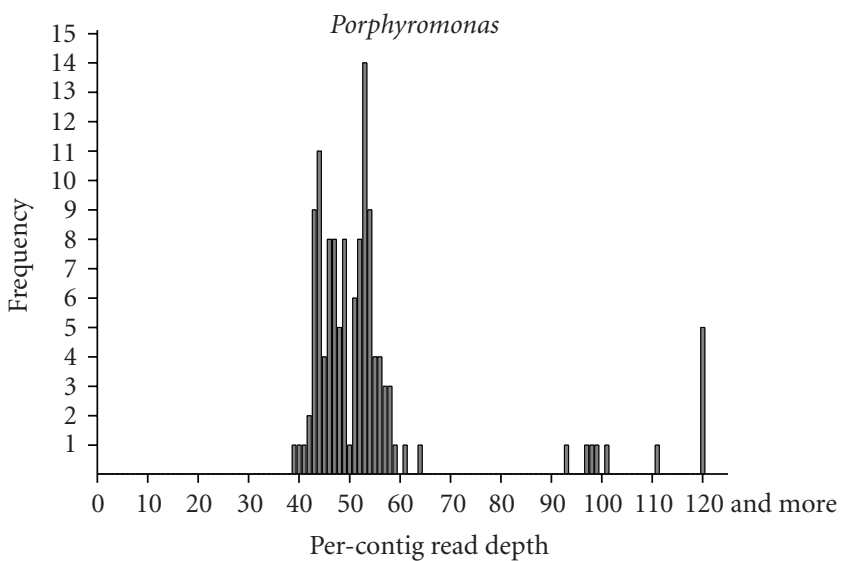

(b)

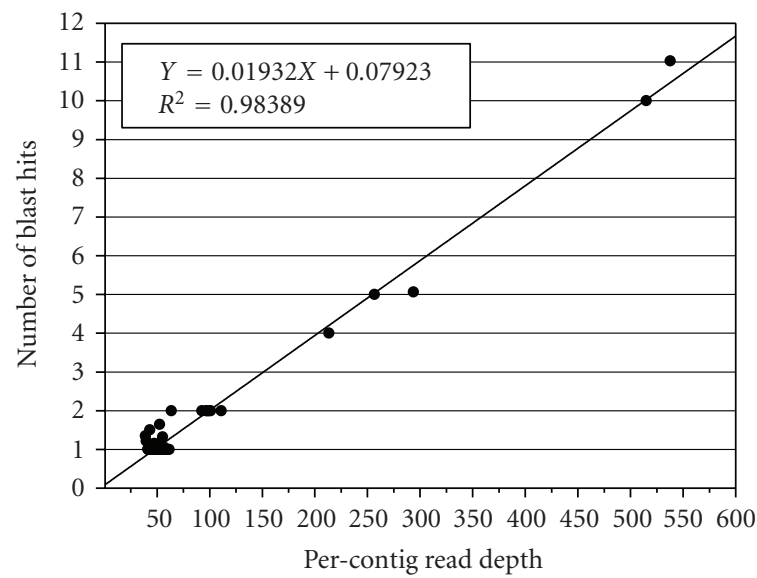

(d)

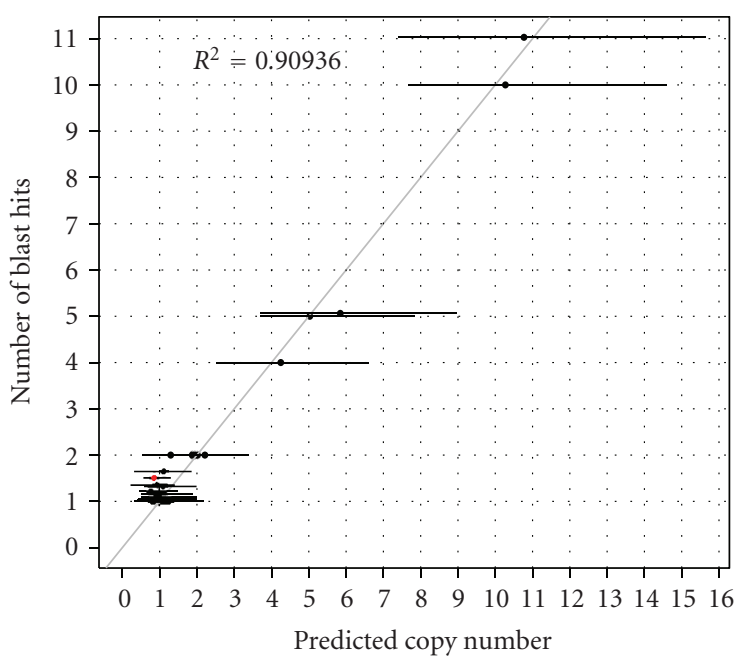

(f)

Figure 1: Correlation between per-contig read depth and genomic copy number. (a) and (b), per-contig read depth frequency distributions for the 454 assemblies for E. coli K12 and P. gingivalis, respectively. (c) and (d): Scatter plot showing the number of BLASTX hits against the reference genome versus the per-contig read depth for each contig for the two assemblies. Linear regression curves are shown. Inset: the result of linear regression analysis. (e) and (f): comparison of the number of BLASTX hits against the reference genome versus the predicted per-contig copy number based on the statistical approach described in the paper. For each contig, the predicted copy number is indicated with the upper and lower confidence intervals. The $1: 1$ line is shown in grey. Copy number estimates with confidence intervals that do not include the known copy number (number of blast hits) are shown in red. Inset: the result of linear regression analysis. 


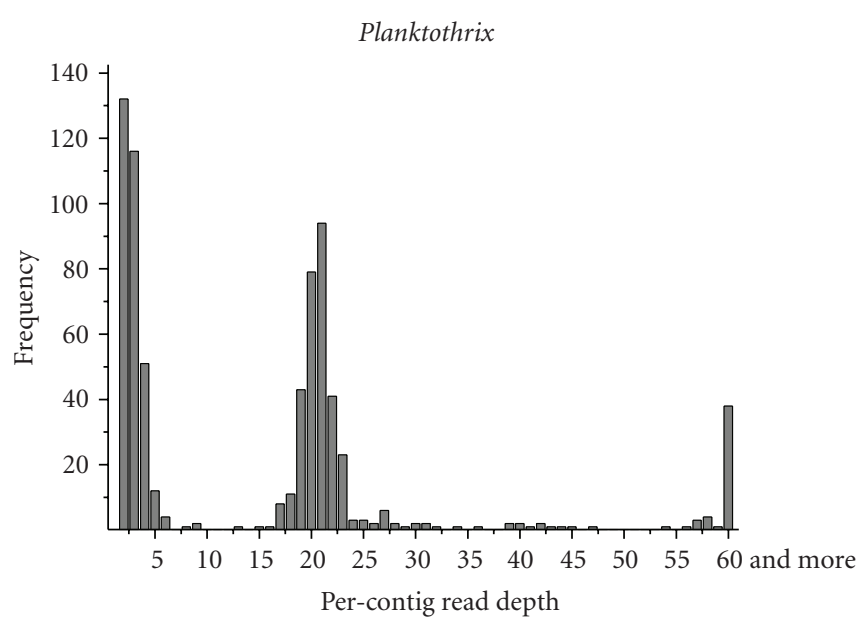

(a)

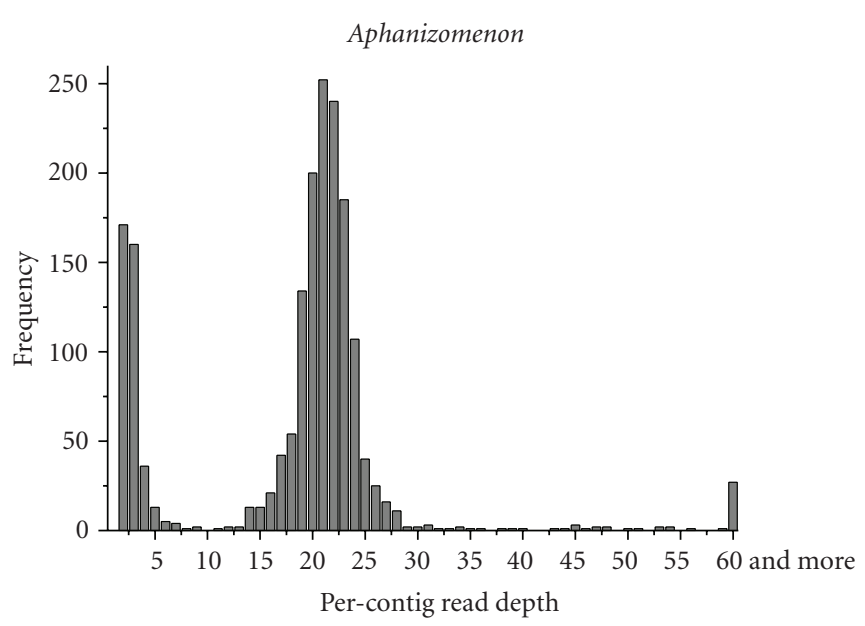

(b)

Figure 2: Per-contig read depth distribution for the cyanobacterial de novo genome assemblies. Per-contig read depth frequency distributions for the P. rubescens NIVA CYA98 (a) and A. flos-aquae 10E6 (b) assemblies.

Table II reports the number of BLAST hits for each contig and the annotations in the regions of the BLAST hits. Many of the regions with multiple copies were annotated as transposons or transposon-related coding sequences. The $5 S, 16 S$, and $23 \mathrm{~S}$ ribosomal RNA genes were also among the high read depth contigs. Transposases and ribosomal RNA regions are known to be present in several copies in bacterial genomes [21, 22].

Next, we tested whether, for a given contig with high read depth, the number of copies present in the genome could be estimated by taking into account the per-contig read depth of all contigs. This would be useful in a situation where no reference genome is available, such as in de novo genome sequencing. To this end, we developed a method for estimating the number of copies present in the genome by assessing the probabilistic relationship between read depth and the number of genomic copies (see Methods). Given truly random sampling of the genome, read depth would follow a Poisson distribution [23], but significant deviations from Poisson assumptions found in real data caused us to employ a more robust methodology.

For both the E. coli and $P$. gingivalis assemblies, we estimated the average oversampling level and the number of copies of each contig present in the genome, together with the upper and lower confidence intervals. The predicted copy numbers were compared to the number of blast hits for each contig for both assemblies, see Figures 1(e) and 1(f), respectively. The copy number estimates and their confidence intervals are also shown in Supplementary Table II. The results show an excellent match between predicted copy number and number of blast hits. Variance and thus confidence intervals increased with mean copy number, which is to be expected in an approximated Poisson process. The method correctly predicted that a contig came from a repeated region (copy number of at least $1.5 \mathrm{x}$ ) of the genome for 17 out of the $18 \mathrm{E}$. coli, and 11 out of 12 $P$. gingivalis contigs, respectively. Therefore, both datasets showed one false negative contig. No single-copy contigs (number of blast hits below 1.5x) had a copy number estimate above $1.5 \mathrm{x}$, therefore there were no false positives. The method therefore had a $100 \%$ specificity for both genomes, and a sensitivity of $94 \%$ for the E. coli, and $92 \%$ for the $P$. gingivalis genomes, respectively. For contigs from repeated regions of the genomes, the actual copy number fell outside the confidence intervals for only $3 \%$ of the E. coli contigs, and none of the $P$. gingivalis contigs, respectively, see Supplementary Table II. It cannot be excluded that this is the result of either a misassembly in the reference genome, a misassembly by Newbler, or an actual copy number variation event in the strain sequenced.

The excellent correspondence between the "known" (based on blast hits) and predicted genomic copy number indicates that the method presented here represents a robust and flexible way to estimate the number of copies for contigs when bacterial genomes or other low-redundancy sequences are sequenced de novo.

3.2. De Novo Assemblies of Cyanobacterial Genomes. A major focus of our research is how different variants of large nonribosomal peptide synthetases (NRPS) and polyketide synthetase gene (PKS) clusters $[24,25]$ evolve in the context of cyanobacterial subpopulation differentiation [26, 27]. To this end, the genomes of the cyanobacterial strains Planktothrix rubescens NIVA CYA98 and Aphanizomenon flos-aquae 10E6 were studied by whole genome shotgun 454 sequencing and the NRPS gene clusters present in both genomes were identified in the assemblies $[4,12]$. In order to determine the repeat content of these genomes, the methods described above were applied to these genomes.

The $P$. rubescens NIVA CYA98 shotgun 454 dataset was assembled, using the 454 Newbler assembler with default settings, into 703 contigs of at least $500 \mathrm{bp}$ [4]. For this assembly, the distribution of the per-contig read depths is shown in Figure 2(a). The distribution showed a large 


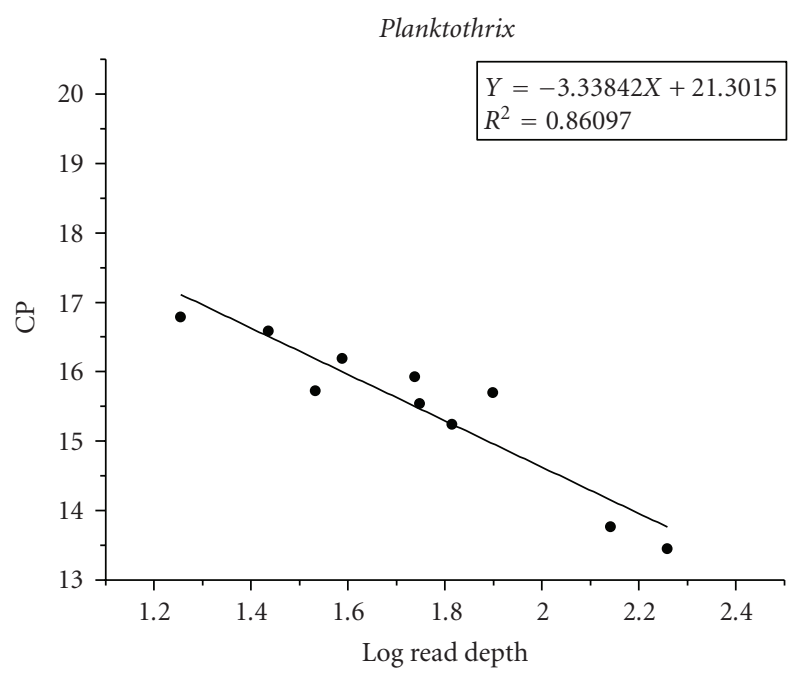

(a)

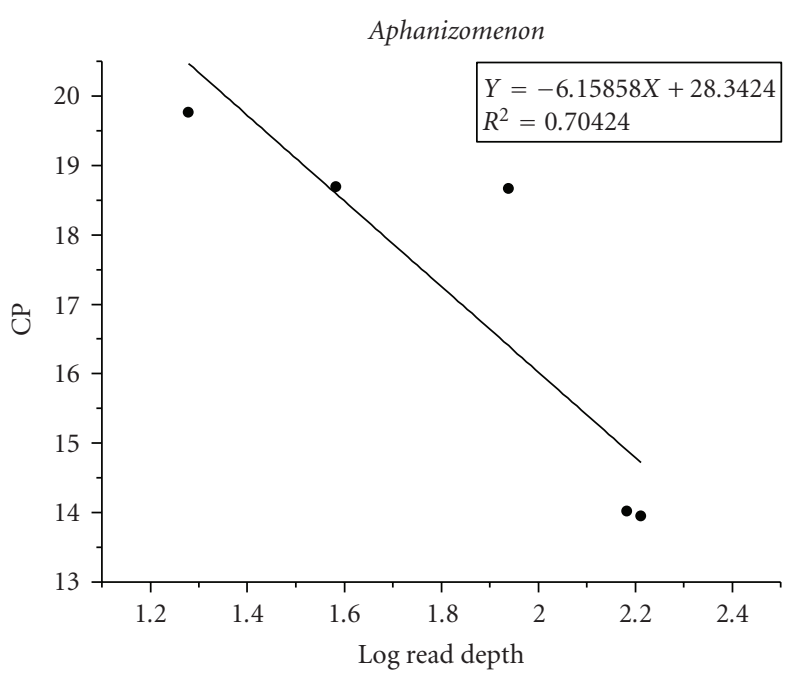

(b)

FIGURE 3: Correlation between read depth and abundance as determined by real-time PCR. Scatter plots of CP values obtained with real-time PCR, versus the logarithm of the local read depth of the region of the PCR primers. (a) P. rubescens assembly (b) A. flos-aquae assembly. Linear regression curves are shown with the regression result in the inset.

peak around a read depth between 2 and $5 \mathrm{x}$ and a second peak between 19 and 23x. However, there were also a number of contigs with a higher read depth, up to $260 x$. We also assembled the reads derived from sequencing a shotgun genomic DNA library from A. flos-aquae 10E6 [12]. The resulting 1811 contigs showed a similar read depth distribution as the $P$. rubescens assembly (Figure 2(b)) with a peak in the distribution from 2 to $5 x$, and a second peak from 17 to $24 \mathrm{x}$. The highest read depth for this assembly was $301 x$.

We hypothesized that contigs with a read depth below 10x were derived from contaminating genomic DNA derived from other bacteria present in the culture, this will be discussed in the next section. Analogously to the E. coli and $P$. gingivalis assemblies, we hypothesized that the peak in the read depth distribution around $20 \mathrm{x}$ represented contigs that had a single copy in the genome, while the remaining, higher read depth contigs were derived from reads stemming from genomic regions present in multiple copies.

To test this latter hypothesis experimentally, real-time PCR primer sets were designed for both cyanobacterial genome assemblies for a selection of contigs with varying degrees of read depth (see Methods). For each species, real-time PCR experiments were performed on the same template for all primer sets. Next, the resulting real-time PCR CP values (CP: Crossing Point, the cycle at which the fluorescence of a sample rises above the background fluorescence; CP values are expected to drop logarithmically with the amount of starting material) were plotted against the log of the local average read depth in the contig at the region of the PCR primers. The results showed high correlation between the CP value and the log of the read depth for both assemblies, with $R$-squared values of 0.861 and 0.704 , respectively (Figure 3 ). This further confirms our assumption that read depth of contigs assembled by Newbler is proportional to the number of copies present in the genome being sequenced.

In order to estimate the genomic copy number of the high read depth contigs for the cyanobacterial assemblies, we used the statistical method developed in this work described above, that is, the empirical, nonparametric approach. We excluded contigs with a read depth below $10 \mathrm{x}$ for this analysis. The results are presented in Supplementary Figure 1. For the $P$. rubescens assembly, 65 out of the 385 contigs (16.9\%) were determined to be from repeated regions (estimated copy number of at least $1.5 \mathrm{x}) ; 16$ (4.2\%) contigs had an estimated copy number over $5 x$, with a maximum of $12.4 \mathrm{x}$. These repeated contigs contained a total of $216,819 \mathrm{bp}$ (3.8\% of the total assembly length). Of the 1419 A. flos-aquae contigs, $55(3.9 \%)$ showed an estimated copy number over $1.5 \mathrm{x}, 11(0.8 \%)$ over $5 \mathrm{x}$ with a maximum of $14.2 \mathrm{x}$. A total of $102,628 \mathrm{bp}$ ( $1.8 \%$ of the total assembly length) was contained in these contigs. Confidence intervals for these estimates are larger than for the E. coli and P. gingivalis assemblies, most likely caused by the fact that the repeated contigs for these assemblies were longer, and thus showed more repeated sequences within each contig or partial contigs being repeated in other places. Table 1 shows the copy number estimates for contigs with an estimated copy number of at least 5. The table also shows the result of comparisons of these contigs against Genbank using BLASTX. For each contig, BLASTX hits are presented in the table. Many high read depth contigs had BLAST hits in transposases, including the four contigs with the highest read depths in both assemblies. Finally, The table also shows the contigs determined by BLASTN searches to contain the $16 \mathrm{~S}$ and $23 \mathrm{~S}$ ribosomal RNAs. Based on the copy number estimates, both the $P$. rubescens and $A$. flos-aquae genomes contain most likely four copies of these rRNAs. Supplementary Table III shows the same results as Table 1, but including all contigs 
TABle 1: Annotation of the high read depths contigs for P. rubescens and A. flos-aquae assemblies. For each contig with an estimated copy number of at least $5 x$, the length, read depth, and estimate of copy number ("Est. copy number") with upper and lower Confidence interval Limits (CL) are shown. In addition, BLASTX results are shown (maximum $E$ value $10^{-16}$ ). When a contig had hits in multiple different regions, these are separated by a comma. The species to which the BLAST hit belongs is shown in between square brackets.

(a) P. rubescens

\begin{tabular}{|c|c|c|c|c|c|c|}
\hline Contig & $\begin{array}{l}\text { Length } \\
\text { (bp) }\end{array}$ & $\begin{array}{l}\text { Read } \\
\text { depth }\end{array}$ & $\begin{array}{l}\text { Est. copy } \\
\text { number }\end{array}$ & $\begin{array}{c}\text { Lower } \\
\text { CL }\end{array}$ & $\begin{array}{l}\text { Upper } \\
\text { CL }\end{array}$ & Features \\
\hline 13664 & 1309 & 259.7 & 12.4 & 9.1 & 19.1 & $\begin{array}{l}\text { transposase, IS4 family protein (Nostoc punctiforme PCC } \\
73102 \text { ) }\end{array}$ \\
\hline 13972 & 937 & 190.3 & 9.2 & 6.5 & 15.1 & transposase, IS4 family protein (Cyanothece sp. PCC 8802) \\
\hline 13823 & 1190 & 180.2 & 8.9 & 6.3 & 12.9 & transposase (Microcystis aeruginosa NIES-843) \\
\hline 13688 & 1109 & 176.3 & 8.8 & 5.8 & 12.9 & transposase (Trichodesmium erythraeum IMS101) \\
\hline 136 & 3509 & 173.3 & 8.6 & 5.6 & 13.7 & No hits \\
\hline 13792 & 9424 & 173.1 & 8.7 & 5.3 & 13.3 & $\begin{array}{l}\text { hypothetical protein Npun_R2618 [Nostoc punctiforme PCC } \\
73102 \text { ], DnaB domain protein helicase domain protein } \\
\text { (Cyanothece sp. PCC 7822) }\end{array}$ \\
\hline 13610 & 852 & 163.2 & 8.0 & 5.2 & 12.6 & No hits \\
\hline 13711 & 1051 & 145.0 & 7.2 & 5.3 & 10.2 & No hits \\
\hline 13735 & 2163 & 144.0 & 7.2 & 4.0 & 11.5 & conserved hypothetical protein (Cyanothece sp. PCC 7425) \\
\hline 13901 & 611 & 140.9 & 7.1 & 4.7 & 9.8 & transposase, IS605 OrfB family (Cyanothece sp. PCC 8801) \\
\hline 13846 & 902 & 132.8 & 7.2 & 2.5 & 9.8 & hypothetical protein L8106_22791 (Lyngbya sp. PCC 8106) \\
\hline 14014 & 770 & 123.8 & 6.0 & 4.3 & 9.8 & Histone-like DNA-binding protein (Lyngbya sp. PCC 8106) \\
\hline 13843 & 1712 & 111.4 & 5.6 & 3.5 & 8.5 & $\begin{array}{l}\text { RNA-directed DNA polymerase (Microcystis aeruginosa } \\
\text { NIES-843) }\end{array}$ \\
\hline 13469 & 669 & 104.2 & 5.2 & 3.8 & 7.4 & No hits \\
\hline 13858 & 641 & 99.5 & 5.1 & 3.0 & 7.2 & hypothetical protein L8106_22631 (Lyngbya sp. PCC 8106) \\
\hline 13921 & 2057 & 99.3 & 5.2 & 1.7 & 9.4 & transposase (Microcystis aeruginosa NIES-843) \\
\hline 13462 & 1575 & 76.9 & 3.7 & 2.5 & 6.0 & $16 \mathrm{~S}$ rRNA \\
\hline 13463 & 2891 & 75.4 & 3.7 & 2.3 & 6.0 & $23 \mathrm{~S}$ rRNA \\
\hline
\end{tabular}

(b) A. flos-aquae

\begin{tabular}{|c|c|c|c|c|c|c|}
\hline Contig & $\begin{array}{l}\text { Length } \\
\text { (bp) }\end{array}$ & $\begin{array}{l}\text { Read } \\
\text { Depth }\end{array}$ & $\begin{array}{l}\text { Est. copy } \\
\text { Number }\end{array}$ & $\begin{array}{c}\text { Lower } \\
\text { CL }\end{array}$ & $\begin{array}{c}\text { Upper } \\
\text { CL }\end{array}$ & Features \\
\hline 13355 & 911 & 301.0 & 14.2 & 9.6 & 24.6 & transposase (Microcystis aeruginosa NIES-843) \\
\hline 13273 & 748 & 257.2 & 12.5 & 6.0 & 25.7 & $\begin{array}{l}\text { transposase (Nodularia spumigena CCY9414), transposase } \\
\text { (Nodularia spumigena CCY9414) }\end{array}$ \\
\hline 13683 & 1256 & 189.6 & 8.6 & 5.5 & 17.7 & transposase (Nostoc sp. PCC 7120) \\
\hline 14262 & 560 & 174.4 & 8.2 & 5.5 & 14.7 & $\begin{array}{l}\text { transposase and inactivated derivatives (Syntrophus } \\
\text { aciditrophicus) }\end{array}$ \\
\hline 14128 & 1185 & 165.6 & 8.0 & 5.0 & 13.5 & $\begin{array}{l}\text { unnamed protein product (Microcystis aeruginosa PCC } \\
7806 \text { ) }\end{array}$ \\
\hline 12918 & 1870 & 163.3 & 7.7 & 4.5 & 14.3 & transposase (Microcystis aeruginosa NIES-843) \\
\hline 525 & 1566 & 160.6 & 7.6 & 4.4 & 13.7 & $\begin{array}{l}\text { hypothetical protein AM1_C0013 (Acaryochloris marina } \\
\text { MBIC11017), hypothetical protein AM1_C0013 } \\
\text { (Acaryochloris marina MBIC11017) }\end{array}$ \\
\hline 214 & 567 & 156.5 & 7.2 & 4.9 & 12.7 & $\begin{array}{l}\text { conserved hypothetical protein (Microscilla marina ATCC } \\
\text { 23134), conserved hypothetical protein (Microscilla marina } \\
\text { ATCC } 23134 \text { ) }\end{array}$ \\
\hline 12934 & 575 & 137.3 & 6.6 & 3.6 & 12.0 & $\begin{array}{l}\text { IS1 transposase subfamily, putative (Synechococcus sp. PCC } \\
7335 \text { ) }\end{array}$ \\
\hline 13740 & 502 & 115.3 & 5.4 & 3.8 & 9.3 & transposase (Cyanothece sp. ATCC 51142) \\
\hline 13286 & 1782 & 109.0 & 6.2 & 0.2 & 14.2 & No hits \\
\hline 13746 & 1585 & 91.0 & 4.3 & 2.3 & 8.1 & $16 \mathrm{~S}$ \\
\hline 13744 & 2169 & 89.9 & 4.2 & 2.7 & 7.8 & $23 \mathrm{~S}$ \\
\hline
\end{tabular}


determined to be from repeated regions (estimated copy number at least $1.5 \mathrm{x}$ ).

The results of our analysis of de novo bacterial genome assemblies using the Newbler assembly program show that read depth-based copy number estimations can be used to de novo identify contigs containing sequences present multiple times in the genome. In addition, we show how contig specific read depth can be used to predict the number of genomic copies of these sequences in the genome, even when no paired read data is available to resolve repeats.

One rationale for sequencing the genome of $P$. rubescens was to identify all NRPS gene clusters putatively present in the genome. When we studied the read depth of the contigs containing these clusters, we concluded that two NRPS gene clusters were present in the genome in multiple copies, while the others were present as single copies [4]. Recombination occurs frequently in NRPS gene clusters (see, $[25,28]$ ) and in cyanobacterial genomes in general [29, 30]. Identifying duplications is not only important in cyanobacterial research, but in most genome projects. The read depth based copy number estimation method presented here will allow us to assess NRPS gene cluster duplications by 454 shotgun sequencing of the relevant strains.

In principle, assessing the relation between read depth and genomic copy number should be possible for all shotgun assemblies based on reads from other next-generation sequencing technologies that do not amplify sequences by propagating in bacteria. Nevertheless, this analysis depends partly on the assembly program; not all programs treat repeats the way the Newbler program does. In addition, read coverage for Illumina/Solexa reads is likely biased towards regions with higher GC content [31, 32]. We therefore expect an analysis of contigs from assemblies using Illumina reads to show a lower correlation between read depth and genomic copy number. Several recent publications describe methods, using next-generation sequencing technology, for determining copy number variations based on read depth $[6,32-38]$. These methods are all based on a well-annotated, usually human, reference genome. Our method aims to provide copy number estimations for de novo assemblies of bacterial genomes.

\subsection{Detection of Contigs of Co-Cultured Bacteria in Non-} Axenic Cyanobacterial Cultures. Both the P. rubescens and A. flos-aquae assemblies showed a large number of contigs with low read depths, between 2 and $5 x$ (Figures 2(a) and 2(b)). We further examined the contigs of these assemblies by plotting the GC percentage of each contig versus the contig read depth for all contigs (Figures $4(\mathrm{a})$ and 4 (b)). The figure shows the contigs with low read depth to the left of the dotted line at 10x read depth and the remaining contigs to the right of this line. For both these assemblies, the contigs with low read depths could be further divided into contigs with a low GC percentage (between $20-25 \%$ and $40-45 \%$ for $P$. rubescens and $A$. flos-aquae, resp.) and a high GC percentage (between $45-50 \%$ and 65-70\%, resp.); see Figures 4(a) and 4(b).
The two groups of contigs, having low (below 10x) and high (above 10x) read depths, were separately compared to the nonredundant NCBI protein database using BLASTX [16], and the top 25 hits were recovered. This analysis was limited to the contigs of at least $500 \mathrm{bp}$ because of the large number of small, low read depth contigs present in the assembly. The program MEGAN [MEtaGenomics Analysis, 17] was used to summarize the results and plot them onto the NCBI taxonomic tree (using a Lowest Common Ancestor algorithm) in a comparative way (Figures $4(\mathrm{c})$ and $4(\mathrm{~d})$ ). The MEGAN results indicated that the contigs with read depths of $10 \mathrm{x}$ and above of both the P. rubescens (Figure 4(c)) and A. flos-aquae (Figure $4(\mathrm{~d})$ ) assemblies were mainly cyanobacterial, with a small fraction of the A. flos-aquae contigs in the Proteobacteria part of the tree. For $P$. rubescens, the contigs with read depths below 10x fell mainly in the phylum Proteobacteria, with some in the genus Bacteroides. For $A$. flos-aquae (Figure 4(d)), the low read depth contigs mapped mainly in the order Flavobacteriales (genus Bacteroides) with the rest determined as Proteobacteria. For both assemblies, the contigs with low read depths and low GC percentage were assigned to the Bacteroides part of the tree, while the Proteobacteria subtree contained the low read depth, high GC percentage contigs.

The contigs with a read depth below 10x were most likely derived from co-cultured bacteria present in the sample used for DNA extraction. It is difficult to obtain axenic cyanobacterial cultures (free of other "contaminating" organisms), and therefore these cultures often contain several different co-cultured bacteria [39, 40, page 139]. The fact that contigs resulting from contaminating reads have a low read depth is a result of the low frequency of the contaminating "genomes": there are relatively few reads present from these genomes (low rate of oversampling), which are assembled in many contigs which have a low read depth. Due to the high oversampling rate of 454 sequencing (typically around 20x or more), the contaminants assemble into contigs with a significantly lower read depth than the contigs of the dominant genome in the sample. Assemblies based on Sanger reads are usually at a lower oversampling ( 7 to $8 \mathrm{x}$ ), preventing the contaminants from being assembled into contigs with clearly lower read depth. In fact, in 454 assemblies of cyanobacterial genomes with a too low oversampling (e.g., below 10x), we could not detect the contaminants based on read depth alone (data not shown).

By removing the contaminating contigs (those with a read depth below 10x) from the assemblies, their statistics improved: for $P$. rubescens, the number of contigs was reduced from the original 703 to 385 (55\%), and the average contig length almost doubled from 8110 to 14280 , and contig N50 length went from 24899 to 25830 (the N50 length is defined such that half of the assembled bases reside in contigs having a length of at least the N50 contig length). For the Aphanizomenon assembly, 1419 of the 1811 (78\%) contigs remained, and the average length increased from 3217 to 3920, and contig N50 length went from 5731 to 5986. Based on the number of reads present in the different contigs, we determined the level of contamination to be around $6 \%$ to $7 \%$ of the reads in the cyanobacterial samples sequenced. 


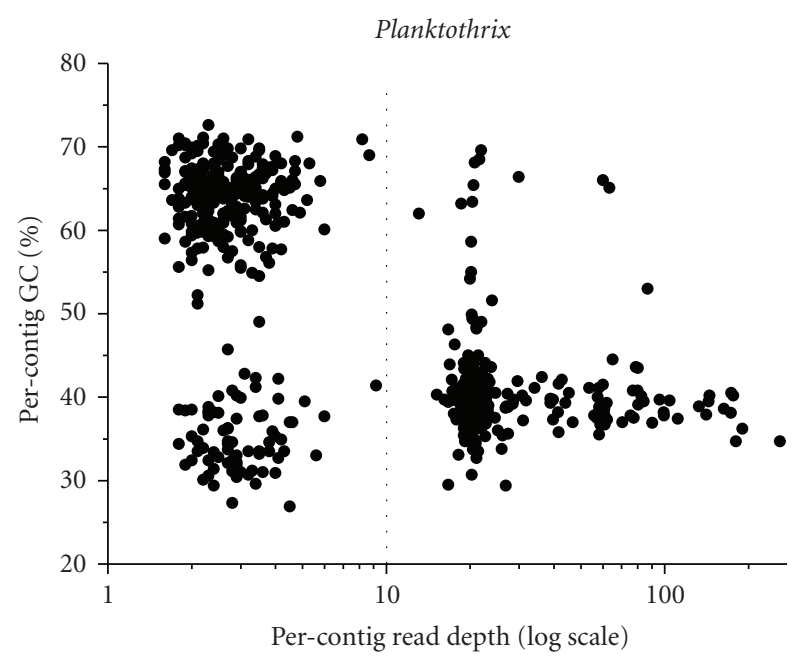

(a)

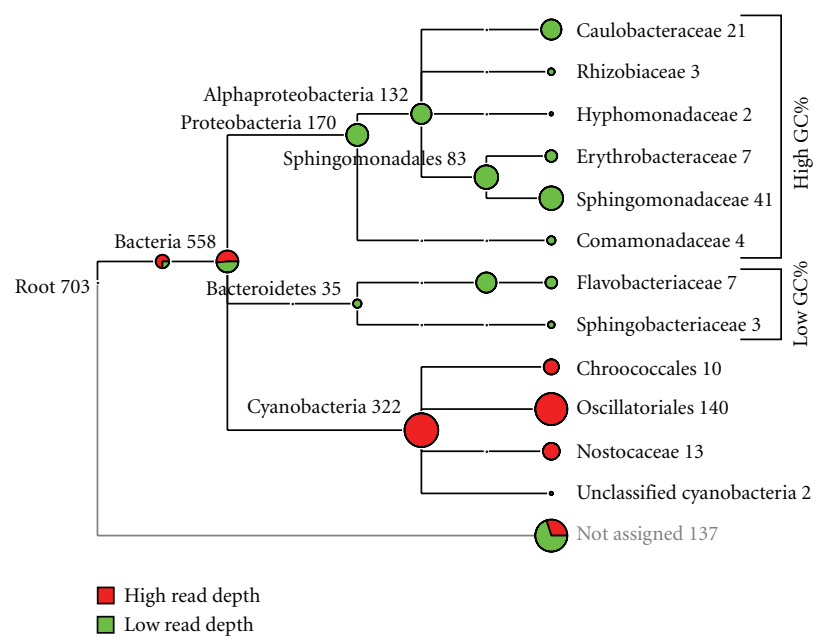

(c)

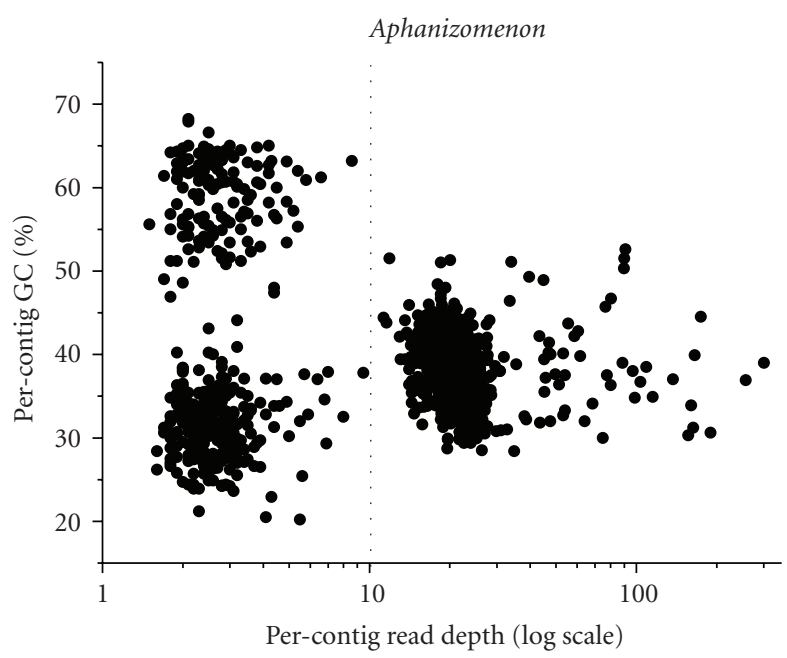

(b)

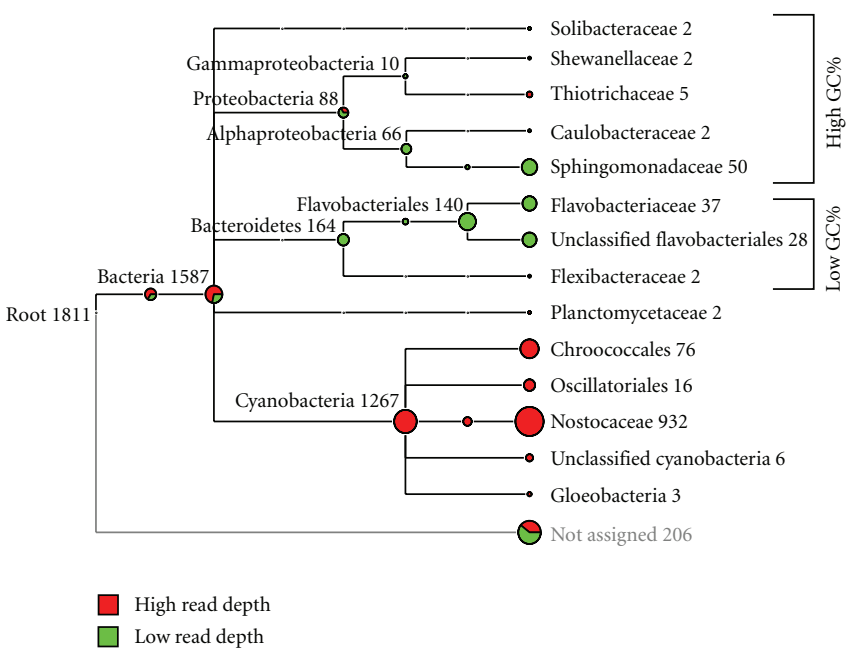

(d)

FIGURE 4: Identifying contigs from contaminating bacteria in cyanobacterial genome assemblies derived from DNA from nonaxenic cultures. (a) and (b): scatter plot showing for each contig (minimum length $500 \mathrm{bp}$ ), the GC percentage and read depth (log scale) for the P. rubescens NIVA CYA98 (a) and A. flos-aquae (b) assemblies. Contigs with low read depths to the left of the dotted line at 10x read depth. The low read depth contigs fall into two clusters based on GC percentage. (c) and (d): MEGAN comparisons of the low (green) and high (red) read depth contigs from the P. rubescens NIVA CYA98 (c) and A. flos-aquae (d) assemblies. Trees collapsed at the Family taxonomic level. Numbers with the taxon names are number of hits summarized to that node and all nodes below in the NCBI taxonomic tree. Circles sizes are log-scale relative to the number of hits. For the contigs with low read depths, it is indicated that if they fall into the high or low GC\% cluster of contigs. "Not assigned": contigs that were not assigned to any branch of the tree due to too low bit score (cutoff at 100) or because they are the only contig that were assigned to a particular taxon.

However, these contaminating reads assembled into $45 \%$ of the contigs in the case of $P$. rubescens and $22 \%$ in the case of A. flos-aquae.

The contigs assembled from these contaminating reads were much shorter than the contigs derived from the remaining set of reads. This is actually a direct cause of the low frequency of the contaminating "genomes": there are relatively few reads present of these genomes (low rate of oversampling), resulting in an assembly consisting of many short contigs with a low read depth.

Our per-contig read depth analysis are thus able to distinguish contigs coming from the co-cultured bacteria from those resulting from the genome of interest. In this way, a straightforward method to obtain a "clean" assembly is to simply select the contigs with a read depth above a certain threshold.

\subsection{Detection of Host Organism Genomic DNA in BAC Assem-} blies. We used our analysis approach based on per-contig read depth on two BAC (Bacterial Artificial Chromosome) shotgun sequencing datasets containing genomic regions from salmon. Close to 75,000 reads for BAC $184 \mathrm{H} 23$ were assembled into 579 contigs of at least $100 \mathrm{bp}$, and just over 55,000 reads into 471 contigs for BAC $114 \mathrm{~L} 13$. 
BAC $184 \mathrm{H} 23$

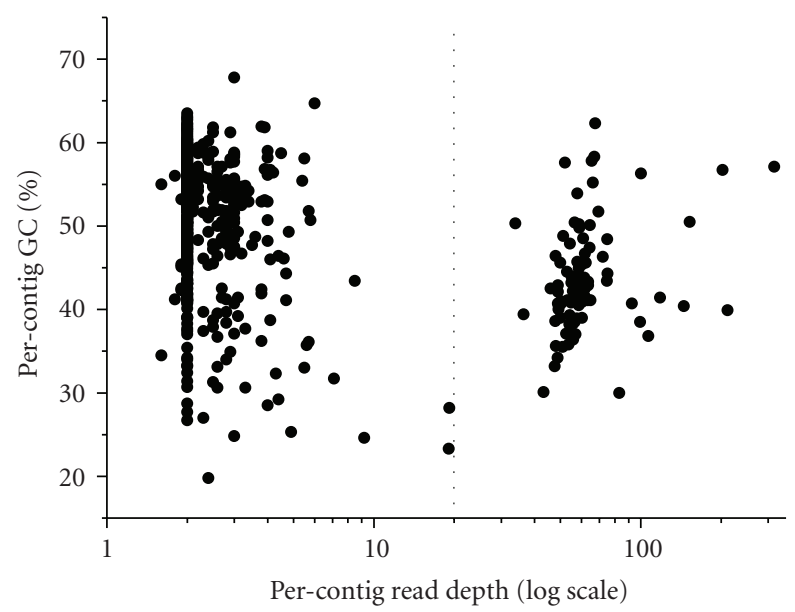

(a)

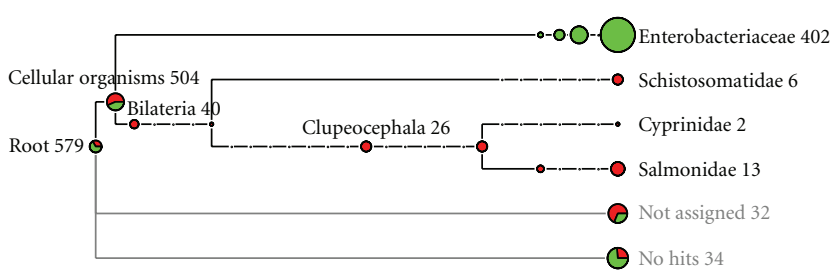

High read depth $\square$ Low read depth

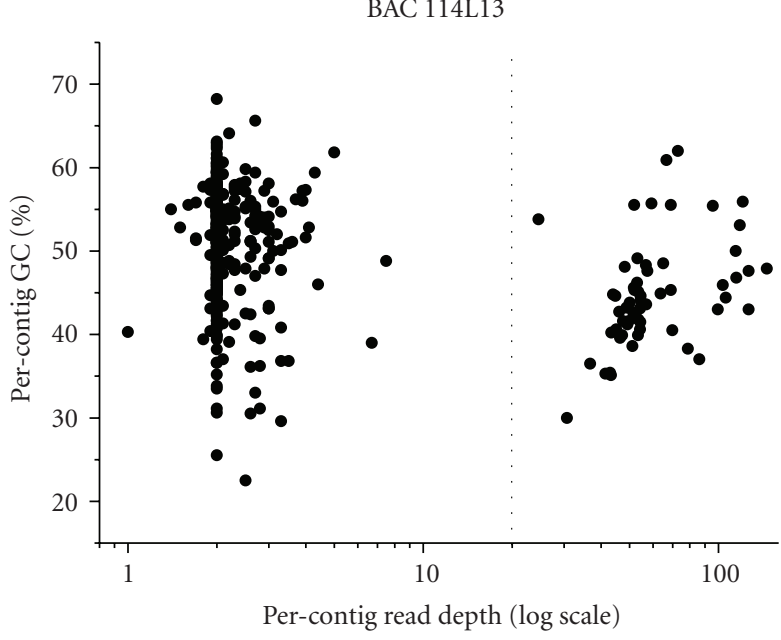

(b)

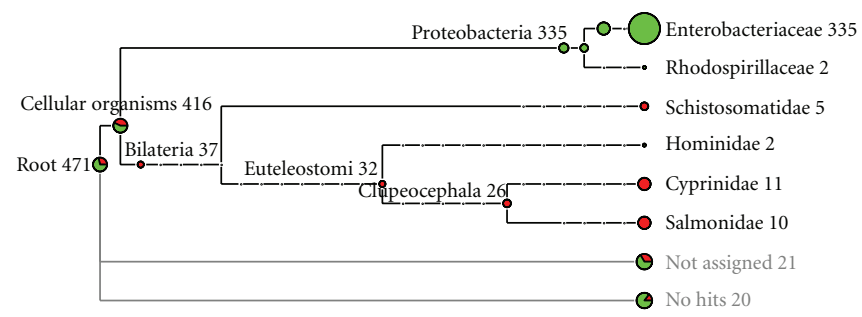

High read depth $\square$ Low read depth

(c)

(d)

FIGURE 5: Identifying bacterial host genomic DNA in BAC assemblies. (a) and (b): scatter plot showing for each contig (minimum length $100 \mathrm{bp}$ ), the GC percentage and read depth (log scale) for Salmon BAC 184H23 (a) and Salmon BAC 114L13 (b) assemblies. Contigs with low read depths to the left of the dotted line at 20x read depth. (c) and (d): MEGAN comparisons of the low and high read depth contigs from the Salmon BAC 184H23 (a) and Salmon BAC 114L13 (b) assemblies. Trees collapsed at the Family level. Contigs with high read depth, in red, cluster into bony fishes (Clupeocephala) with a few hits classified as Schistosomatidae (flatworms). Numbers with the taxon names are number of hits summarized to that node and all nodes below in the NCBI taxonomic tree. Circles sizes are log-scale relative to the number of hits. "Not assigned": contigs that were not assigned to any branch of the tree due to too low bit score (cutoff at 30 ) or because they are the only contig that were assigned to a particular taxon.

Figures 5(a) and 5(b) show the GC percentage versus read depth plots for the assemblies of these BACs. As for the cyanobacterial datasets, two clusters of contigs could be observed for these assemblies, those with low read depths (below 20x) and those with high read depths (above $20 \mathrm{x})$. Comparison of the BAC assembly contigs with the NCBI nonredundant protein database using BLASTX, and MEGAN analysis of the resulting hits (shown in Figures 5(c) and 5(d)) identified contigs with low read depths as coming from bacteria, that is, Enterobacteriaceae (to which Escherichia coli belongs). This indicated that the contigs with low read depth were derived from host genomic DNA that was copurified during BAC DNA sample preparation. The level of host contaminating reads was around $2 \%$ for the BAC datasets, however, they assembled into more than $85 \%$ of the contigs. The high read depths contigs of both BACs came exclusively from eukaryotes, mainly from Clupeocephala, (a group of bony fishes including salmon). A few BLASTX hits were assigned to Schistosomatidae (parasitic flatworms), but these contigs had both fish and Schistosomatidae as best BLASTX hits. The flatworm hits may result from the fact that Schistosomatidae worms can have fish as their host and have been shown to take up host specific genes in their genome [41].

In the case of BAC assemblies (or other sequences propagated in bacteria) where the genome sequence of the host is known, one could remove the contaminating reads with the help of read filtering against the host genome sequence, or BLAST analysis of contigs against the reference genome. We provide an alternative filtering method, based on cleaning up the assembly by using a minimum read depth cutoff.

The approach of using contig read depth selection has been described in one instance before. It was shown that for a 454 assembly of Sulcia muelleri [42], an insect symbiont, the genomic DNA prepared to perform 454 sequencing was contaminated with genomic DNA of the host, and of a second symbiont, Baumannia. Of the total of 416 contigs 
assembled, a selection was made based on average depth [42, supplementary FigureS1], leading to 25 high read depth contigs, of which 23 were further assembled into the complete genome of $S$. Muelleri.

\section{Conclusions}

Contigs made from 454 reads using the Newbler assembler have a read depth that is linearly correlated to the number of copies present in the genome. Bacterial genome assemblies therefore show a number of contigs with higher read depths that represent repeated genomic regions (e.g., transposons, ribosomal RNA genes). We present a robust statistical method to de novo estimate the number of copies of these contig sequences present in the genome based on the contig specific read depth. Most importantly, analysis of contigspecific read depth will extend the amount of information that can be gained from a de novo 454 shotgun assembly using Newbler.

In addition, our per-contig read depth analysis is useful to detect and remove contigs assembled from reads derived from contaminating DNA, since contaminant contigs are likely to have low read depths. This is particularly useful when the sequence of the contaminating genome or genomes is not available, such as in the case of reads obtained from impure (nonaxenic) cultures. There are many other possible examples of contaminated read datasets, such as human viruses derived from cell cultures leading to human DNA contamination, and parasite DNA preparations containing host DNA. We expect contig selection based on read depth to be an essential tool for "cleaning up" contaminated 454 shotgun sequencing read assemblies in future studies.

\section{Acknowledgments}

The authors would like to thank Anke Stüken for allowing them to use the Aphanizomenon dataset, and Unni Grimholt for the BAC datasets. Ave Tooming-Klunderud is acknowledged for expert 454 sequencing, and Tom Kristensen and Bastiaan Star for critical reading of the manuscript. This work was supported by the Norwegian Research Council through grants from the programs AVIT, FUGE, and GenoFisk, respectively.

\section{References}

[1] T. Wicker, E. Schlagenhauf, A. Graner, T. J. Close, B. Keller, and N. Stein, "454 sequencing put to the test using the complex genome of barley," BMC Genomics, vol. 7, article 275, 2006.

[2] M. Pop and S. L. Salzberg, "Bioinformatics challenges of new sequencing technology," Trends in Genetics, vol. 24, no. 3, pp. 142-149, 2008.

[3] Phrap, http://www.phrap.org/.

[4] T. B. Rounge, T. Rohrlack, A. J. Nederbragt, T. Kristensen, and K. S. Jakobsen, "A genome-wide analysis of nonribosomal peptide synthetase gene clusters and their peptides in a Planktothrix rubescens strain," BMC Genomics, vol. 10, no. 1, article 396, 2009.
[5] 454 Case Study: Genome Coverage of Neurospora crassa, http://www.454.com/downloads/454_CASE_STUDY_ genome_coverage.pdf.

[6] K. Swaminathan, K. Varala, and M. E. Hudson, "Global repeat discovery and estimation of genomic copy number in a large, complex genome using a high-throughput 454 sequence survey," BMC Genomics, vol. 8, article 132, 2007.

[7] D. A. Wheeler, M. Srinivasan, M. Egholm, et al., "The complete genome of an individual by massively parallel DNA sequencing," Nature, vol. 452, no. 7189, pp. 872-876, 2008.

[8] J.-M. Aury, C. Cruaud, V. Barbe, et al., "High quality draft sequences for prokaryotic genomes using a mix of new sequencing technologies," BMC Genomics, vol. 9, article 603, 2008.

[9] M. Riley, T. Abe, M. B. Arnaud, et al., "Escherichia coli K-12: a cooperatively developed annotation snapshot-2005," Nucleic Acids Research, vol. 34, no. 1, pp. 1-9, 2006.

[10] The NCBI Short Read Archive, http://www.ncbi.nlm .nih.gov/Traces/sra/sra.cgi.

[11] K. E. Nelson, R. D. Fleischmann, R. T. DeBoy, et al., "Complete genome sequence of the oral pathogenic Bacterium Porphyromonas gingivalis strain W83," Journal of Bacteriology, vol. 185, no. 18, pp. 5591-5601, 2003.

[12] A. Stüken, A. J. Nederbragt, and K. S. Jakobsen, "Cylindrospermopsin biosynthesis cluster in Aphanizomenon flos-aquae," submitted for publication.

[13] M. Margulies, M. Egholm, W. E. Altman, et al., "Genome sequencing in microfabricated high-density picolitre reactors," Nature, vol. 437, no. 7057, pp. 376-380, 2005.

[14] R Development Core Team, R: A Language and Environment for Statistical Computing, R Foundation for Statistical Computing, Vienna, Austria, 2008.

[15] Primer3, http://primer3.sourceforge.net/.

[16] S. F. Altschul, T. L. Madden, A. A. Schäffer, et al., "Gapped BLAST and PSI-BLAST: a new generation of protein database search programs," Nucleic Acids Research, vol. 25, no. 17, pp. 3389-3402, 1997.

[17] D. H. Huson, A. F. Auch, J. Qi, and S. C. Schuster, "MEGAN analysis of metagenomic data," Genome Research, vol. 17, no. 3, pp. 377-386, 2007.

[18] F. R. Blattner, G. Plunkett III, C. A. Bloch, et al., "The complete genome sequence of Escherichia coli K-12," Science, vol. 277, no. 5331, pp. 1453-1462, 1997.

[19] S. L. Chissoe, M. A. Marra, L. Hillier, R. Brinkman, R. K. Wilson, and R. H. Waterston, "Representation of cloned genomic sequences in two sequencing vectors: correlation of DNA sequence and subclone distribution," Nucleic Acids Research, vol. 25, no. 15, pp. 2960-2966, 1997.

[20] J. A. Bailey, Z. Gu, R. A. Clark, et al., "Recent segmental duplications in the human genome," Science, vol. 297, no. 5583, pp. 1003-1007, 2002.

[21] P. Siguier, J. Filee, and M. Chandler, "Insertion sequences in prokaryotic genomes," Current Opinion in Microbiology, vol. 9, no. 5, pp. 526-531, 2006.

[22] Z. M.-P. Lee, C. Bussema III, and T. M. Schmidt, " $r r n \mathrm{DB}$ : documenting the number of rRNA and tRNA genes in bacteria and archaea," Nucleic Acids Research, vol. 37, supplement 1, pp. D489-D493, 2009.

[23] E. S. Lander and M. S. Waterman, "Genomic mapping by fingerprinting random clones: a mathematical analysis," Genomics, vol. 2, no. 3, pp. 231-239, 1988.

[24] A. Tooming-Klunderud, T. Rohrlack, K. Shalchian-Tabrizi, T. Kristensen, and K. S. Jakobsen, "Structural analysis of a nonribosomal halogenated cyclic peptide and its putative operon 
from Microcystis: implications for evolution of cyanopeptolins," Microbiology, vol. 153, no. 5, pp. 1382-1393, 2007.

[25] B. Mikalsen, G. Boison, O. M. Skulberg, et al., "Natural variation in the microcystin synthetase operon mcyABC and impact on microcystin production in Microcystis strains," Journal of Bacteriology, vol. 185, no. 9, pp. 2774-2785, 2003.

[26] T. Rohrlack, B. Edvardsen, R. Skulberg, et al., "Oligopeptide chemotypes of the toxic freshwater cyanobacterium Planktothrix can form subpopulations with dissimilar ecological traits," Limnology and Oceanography, vol. 53, no. 4, pp. 12791293, 2008.

[27] T. B. Rounge, T. Rohrlack, B. Decenciere, B. Edvardsen, T. Kristensen, and K. S. Jakobsen, "Subpopulation differentiation associated with nonribosomal peptide synthetase gene cluster dynamics in the cyanobacterium Planktothrix," the Journal of Phycology, in press.

[28] Y. Tanabe, K. Kaya, and M. M. Watanabe, "Evidence for recombination in the microcystin synthetase (mcy) genes of toxic cyanobacteria Microcystis spp," Journal of Molecular Evolution, vol. 58, no. 6, pp. 633-641, 2004.

[29] O. Zhaxybayeva, J. P. Gogarten, R. L. Charlebois, W. F. Doolittle, and R. T. Papke, "Phylogenetic analyses of cyanobacterial genomes: quantification of horizontal gene transfer events," Genome Research, vol. 16, no. 9, pp. 1099-1108, 2006.

[30] K. Rudi, O. M. Skulberg, and K. S. Jakobsen, "Evolution of cyanobacteria by exchange of genetic material among phyletically related strains," Journal of Bacteriology, vol. 180, no. 13, pp. 3453-3461, 1998.

[31] J. C. Dohm, C. Lottaz, T. Borodina, and H. Himmelbauer, "Substantial biases in ultra-short read data sets from highthroughput DNA sequencing," Nucleic Acids Research, vol. 36, no. 16, p. e105, 2008.

[32] D. Y. Chiang, G. Getz, D. B. Jaffe, et al., "High-resolution mapping of copy-number alterations with massively parallel sequencing," Nature Methods, vol. 6, no. 1, pp. 99-103, 2009.

[33] E. Arner, E. Kindlund, D. Nilsson, et al., "Database of Trypanosoma cruzi repeated genes: 20000 additional gene variants," BMC Genomics, vol. 8, article 391, 2007.

[34] C. Alkan, J. M. Kidd, T. Marques-Bonet, et al., "Personalized copy number and segmental duplication maps using nextgeneration sequencing," Nature Genetics, vol. 41, no. 10, pp. 1061-1067, 2009.

[35] S. Yoon, Z. Xuan, V. Makarov, K. Ye, and J. Sebat, "Sensitive and accurate detection of copy number variants using read depth of coverage," Genome Research, vol. 19, no. 9, pp. 1586$1592,2009$.

[36] B. Daines, H. Wang, Y. Li, Y. Han, R. Gibbs, and R. Chen, "High-throughput multiplex sequencing to discover copy number variants in Drosophila," Genetics, vol. 182, no. 4, pp. 935-941, 2009.

[37] C. Xie and M. T. Tammi, "CNV-seq, a new method to detect copy number variation using high-throughput sequencing," BMC Bioinformatics, vol. 10, article 80, 2009.

[38] J. Macas, P. Neumann, and A. Navratilova, "Repetitive DNA in the pea (Pisum sativum L.) genome: comprehensive characterization using 454 sequencing and comparison to soybean and Medicago truncatula," BMC Genomics, vol. 8, article 427, 2007.

[39] M. J. Ferris and C. F. Hirsch, "Method for isolation and purification of cyanobacteria," Applied and Environmental Microbiology, vol. 57, no. 5, pp. 1448-1452, 1991.

[40] H. W. Paerl, "Marine plankton," in The Ecology of Cyanobacteria Their Diversity in Time and Space, B. Whitton and M. Potts, Eds., pp. 121-148, Springer, New York, NY, USA, 2000.
[41] F. Liu, J. Lu, W. Hu, et al., "New perspectives on host-parasite interplay by comparative transcriptomic and proteomic analyses of Schistosoma japonicum," PLoS Pathogens, vol. 2, no. 4, p. e29, 2006.

[42] J. P. McCutcheon and N. A. Moran, "Parallel genomic evolution and metabolic interdependence in an ancient symbiosis," Proceedings of the National Academy of Sciences of the United States of America, vol. 104, no. 49, pp. 19392-19397, 2007. 


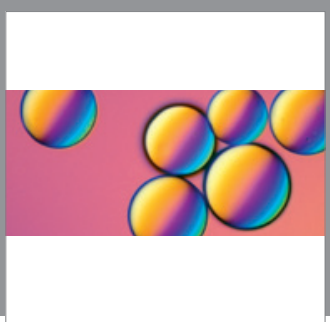

Peptides

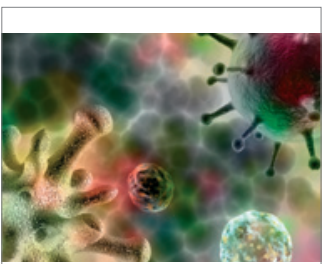

\section{Virology}

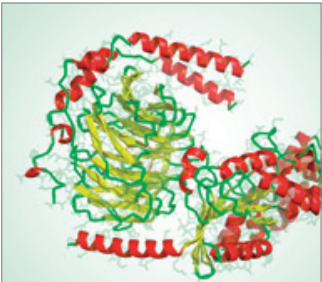

Signal Transduction
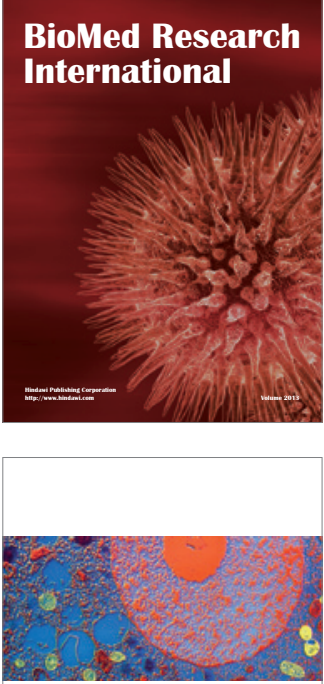

ISRN

Cell Biology
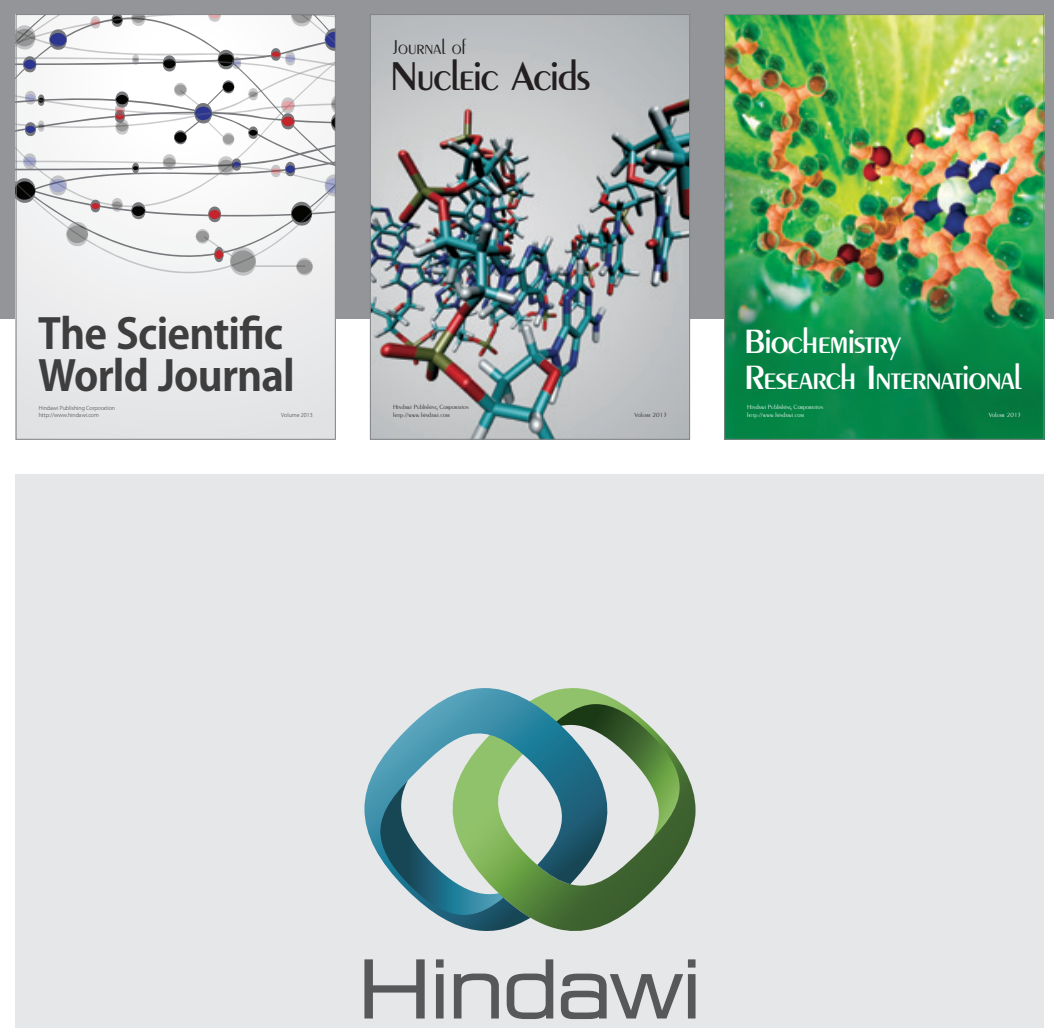

Submit your manuscripts at http://www.hindawi.com

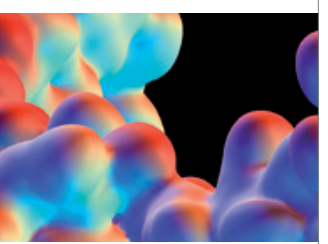

Enzyme Research

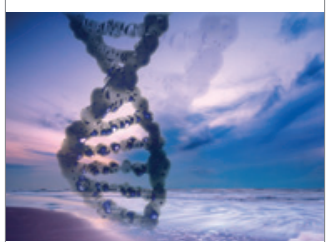

Evolutionary Biology
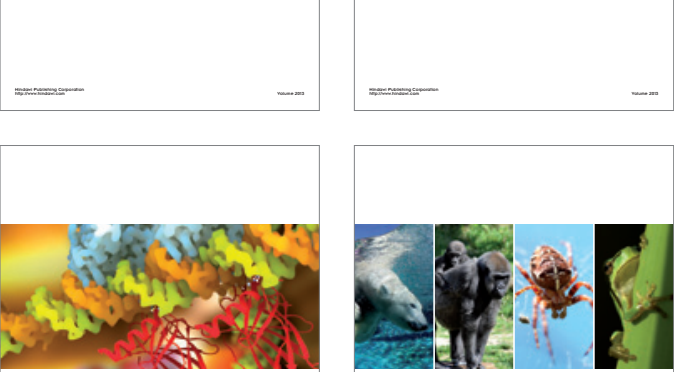

ISRN

Molecular Biology

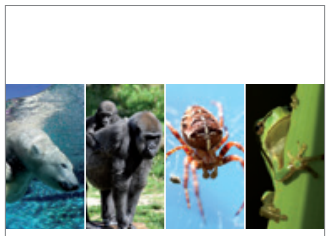

ISRN Zoology

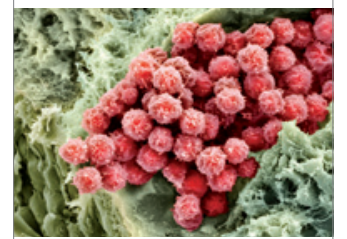

Stem Cells International

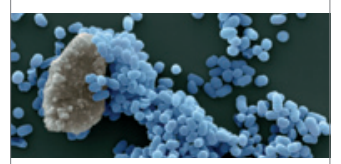

ISRN

Biotechnology
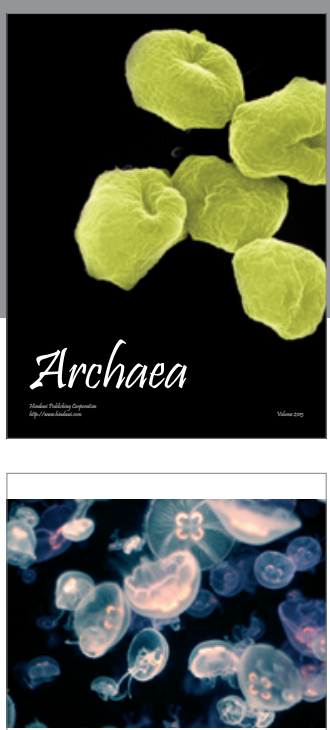

Journal of Marine Biology
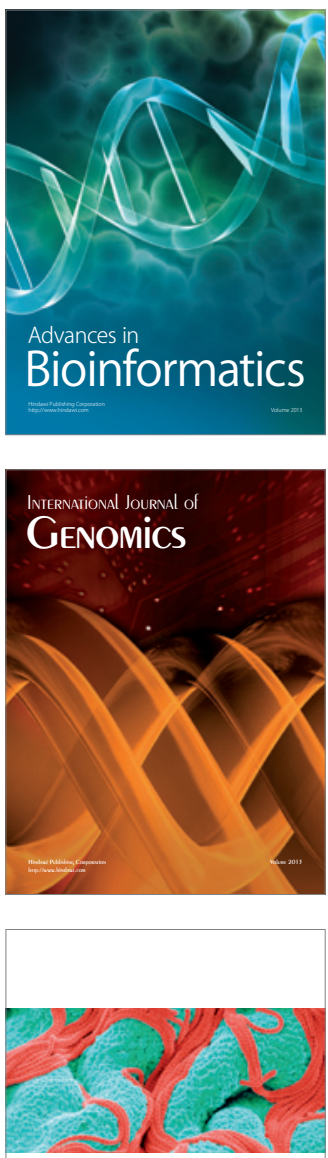

ISRN

Microbiology 